\title{
CLOSED DENSELY DEFINED OPERATORS COMMUTING WITH MULTIPLICATIONS IN A MULTIPLIER PAIR
}

\author{
DON HADWIN, ZHE LIU, AND ERIC NORDGREN
}

(Communicated by Richard Rochberg)

Dedicated to the memory of Bill Arveson, an inspiration to us all

\begin{abstract}
For a multiplier pair $(X, Y)$ we study the closed densely defined operators $T$ on $X$ that commute with all of the multiplications by right multipliers in $X$. We apply our general results to special cases involving $H^{p}$, completions of $L^{\infty}[0,1]$ with respect to certain norms, and the completion of a $I I_{1}$ factor von Neumann algebra with respect to a unitarily invariant norm, where we show that each such $T$ is a "left multiplication". However, we give an example of a closed densely defined operator on the Bergman space that commutes with all multiplications by $H^{\infty}$-functions but is not a multiplication operator.
\end{abstract}

\section{INTRODUCTION}

In [6, 7] the first and third authors introduced and studied the notion of a multiplier pair $(X, Y)$, where $X$ is a Banach space that is a vector subspace of a Hausdorff topological vector space $Y$ with a separately continuous bilinear map (multiplication) $\cdot: X \times X \rightarrow Y$ such that the set $\mathcal{L}_{0}=\{x \in X: x \cdot X \subseteq X\}$ of left multipliers and the set $\mathcal{R}_{0}=\{x \in X: X \cdot x \subseteq X\}$ of right multipliers are dense in $X$, and such that there are dense subsets $\mathcal{E} \subseteq \mathcal{L}_{0}, \mathcal{F} \subseteq X, \mathcal{G} \subseteq \mathcal{R}_{0}$ such that

$$
(a \cdot b) \cdot c=a \cdot(b \cdot c)
$$

whenever $a \in \mathcal{E}, b \in \mathcal{F}, c \in \mathcal{G}$. Moreover, there is an $e \in X$ such that $e \cdot x=x \cdot e=x$ for every $x \in X$. It was shown that, for each $x \in X$, the linear transformations $R_{x}$ and $L_{x}$ defined by

$$
L_{x} a=x \cdot a, R_{x} a=a \cdot x
$$

are closed densely defined operators on $X$. Moreover, $L_{x}$ is bounded on $\mathcal{R}_{0}$ if and only if $x \in \mathcal{L}_{0}$ and $R_{x}$ is bounded on $\mathcal{L}_{0}$ if and only if $x \in \mathcal{R}_{0}$. Thus $\mathcal{R}=$ $\left\{R_{x}: x \in \mathcal{R}_{0}\right\}$ and $\mathcal{L}=\left\{L_{x}: x \in \mathcal{L}_{0}\right\}$ are unital subalgebras of $B(X)$ (the set of bounded linear operators on $X$ ). It was proved in [6] that $\mathcal{L}$ and $\mathcal{R}$ are each other's commutant, which implies that $\mathcal{L}$ and $\mathcal{R}$ are closed in the weak operator topology. In the setting of multiplier pairs a general notion of a composition operator was defined.

Received by the editors November 11, 2011.

2010 Mathematics Subject Classification. Primary 46B99, 47B47; Secondary 30H10, 30H15, $46 \mathrm{~L} 10$.

Key words and phrases. Multiplier pair, closed operator, $I I_{1}$-factor, Hardy space. 
Suppose $B(W)$ denotes the Banach algebra of all bounded linear transformations on a Banach space $W$, and suppose $\mathcal{A}, \mathcal{B}$ are unital Banach subalgebras of $B(W)$ such that $\mathcal{B}^{\prime}=\mathcal{A}$ and $\mathcal{A}^{\prime}=\mathcal{B}$, where $\mathcal{S}^{\prime}$ denotes the commutant of a set of operators $\mathcal{S}$, i.e., the set of operators commuting with every element of $\mathcal{S}$. Suppose $T$ is a linear transformation whose domain $\mathcal{D}(T)$ and range $\mathcal{R}(T)$ are linear subspaces of $W$. We say that $T$ commutes with $\mathcal{B}$ (or $T$ is affiliated with $\mathcal{A}$ ) if, for every $S \in \mathcal{B}$, we have $S(\mathcal{D}(T)) \subseteq \mathcal{D}(T)$ and, for every $x \in \mathcal{D}(T)$, we have $S T x=T S x$. This is equivalent to saying that, for every invertible $S \in \mathcal{B}$, we have $S T=T S$. It is also equivalent to saying that, for every $S \in \mathcal{B}$,

$$
(S \oplus S) \operatorname{Graph}(T) \subseteq \operatorname{Graph}(T),
$$

where $\operatorname{Graph}(T)=\{(x, y) \in W \times W: y=T x\}$ is the graph of $T$. It easily follows that if $\operatorname{Graph}(T)$ is closed, then the set

$$
\mathcal{S}=\{S \in B(W): S(\mathcal{D}(T)) \subseteq \mathcal{D}(T), \forall x \in \mathcal{D}(T) S T x=T S x\}
$$

is a unital algebra that is closed in the weak operator topology. In order to show that $\mathcal{B} \subseteq \mathcal{S}$, it is sufficient to show that $\mathcal{S}$ contains a set of operators $\mathcal{B}_{0}$ such that the unital weak operator closed algebra generated by $\mathcal{B}_{0}$ is $\mathcal{B}$.

In this paper we study the problem of determining for a multiplier pair $(X, Y)$ the closed densely defined operators $T$ on $X$ that commute with $\mathcal{R}$ (i.e., are affiliated with $\mathcal{L}$ ). The symmetry of the situation makes this problem "equivalent" to that of finding the closed densely defined operators on $X$ that commute with $\mathcal{L}$ (i.e., are affiliated with $\mathcal{R}$ ).

In [6] it was shown that, for every $x \in X, L_{x}$ commutes with every operator in $\mathcal{R}$ and $R_{x}$ commutes with every operator in $\mathcal{L}$. In general, we expect that the closed densely defined operators commuting with $\mathcal{R}$ should be left multiplications of some sort. We will prove general results that affirm this notion in a large number of cases, but, when $X$ is the Bergman space on the unit disk, we construct a closed densely defined operator commuting with $\mathcal{L}=\mathcal{R}=H^{\infty}$ that is not a multiplication by any function.

Examples of this problem have been studied by D. Suárez [18] and S. Seubert 17] in the case of the algebra generated by the unilateral shift operator, by D. Sarason [13] in the case of a restricted shift operator [14, [15, and by H. Bercovici [1] in the case of a $C_{0}$-contraction. A result for von Neumann algebras was proved by Nelson [11. The problem for $L^{\infty}[0,1]$ acting on $L^{2}[0,1]$ was discussed in $[9$.

In [6] many examples of multiplier pairs were constructed. Many of them satisfy more conditions than assumed in the definition. We define a multiplier pair $(X, Y)$ to be a special multiplier pair if the multiplication $\cdot: Y \times Y \rightarrow Y$ is defined and separately continuous and $(Y,+, \cdot)$ is a ring with identity $e$.

\section{General Results}

We begin with algebraic results that will apply to special multiplier pairs. If $\mathcal{R}$ is a ring and $\mathcal{S} \subseteq \mathcal{R}$, we say that $\mathcal{S}$ is left-separating if, for every $x \in \mathcal{R}, x \cdot \mathcal{S}=\{0\}$ implies $x=0$.

Theorem 1. Suppose $1 \in Y$ is a ring, $1 \in \mathcal{R}$ is a subring of $Y$ and $\mathcal{R} \subset X \subset Y$, where $X$ is a right $\mathcal{R}$-module. Suppose $G \subset X \times X$ is a graph and a right $\mathcal{R}$-module 
containing an element $(g, h)$ such that

(1) $g$ has a left-inverse $g^{-1}$ in $Y$,

(2) for every $x \in X,\{u \in \mathcal{R}: \exists v \in \mathcal{R}$ such that $x u=g v\}$ is left-separating for $Y$.

Then $G \subseteq \operatorname{Graph}\left(L_{h g^{-1}}\right)$.

Proof. Suppose $(x, y) \in G, u, v \in \mathcal{R}$ and $x u=g v$. Then $(x, y) u-(g, h) v=$ $(0, y u-h v) \in G$. Since $(0,0) \in G$ and $G$ is a graph, we know $y u-h v=0$. Thus $y u=h v=h g^{-1}(g v)=h g^{-1}(x u)$. Hence, for every $u \in \mathcal{R}$ for which there is a $v \in \mathcal{R}$ such that $x u=g v$, we have

$$
\left[y-L_{h g^{-1}} x\right] u=0 .
$$

It follows from (2) that $y=L_{h g^{-1}} x$ for every $(x, y) \in G$.

If $R$ is an integral domain, the field of fractions $\hat{R}$ of $R$ is the field of formal quotients $\frac{a}{b}$ with $a, b \in R$ and the natural addition and multiplication. We think of $R \subseteq \hat{R}$ by identifying $x \in R$ with $\frac{x}{1} \in \hat{R}$.

Corollary 1. Suppose $F$ is the field of fractions of an integral domain $R$, and $R \subset X \subset F$ and $X$ is an $R$-module. Suppose $G \subset X \times X$ is a graph and an $R$-module. Then there is a $\varphi \in F$ such that $G$ is contained in the graph of $y=\varphi x$ in $F \times F$.

Corollary 2. Suppose $(X, Y)$ is a special multiplier pair such that $Y$ is an integral domain and let $\hat{Y}$ denote the field of quotients over $Y$. Suppose also that $X \subseteq \hat{\mathcal{R}}_{0}$. If $G \subseteq X \times X$ is a graph that is an $\mathcal{R}_{0}$-module, then there is a $\varphi \in \hat{\mathcal{R}}_{0}$ such that $G \subseteq \operatorname{Graph}\left(L_{\varphi}\right)$. Moreover, if the domain of $G$ is dense in $X$ and $\tau: Y \rightarrow \mathbb{C}$ is a unital algebra homomorphism whose restriction to $X$ is continuous, then there are $f, g \in \mathcal{R}_{0}$ such that $\varphi=g / f$ and $\tau(f) \neq 0$.

Proof. The first part follows immediately from Theorem 1. Next, suppose the domain of $G$ is dense and $\tau: Y \rightarrow \mathbb{C}$ is a multiplicative linear functional whose restriction to $X$ is continuous. It follows that the domain of $G$ cannot be contained in $\operatorname{ker} \tau$. Hence there is an $(f, g) \in G$ such that $\tau(f) \neq 0$. Since $g=\varphi f$, we have that $\varphi=g / f$ and $\tau(f) \neq 0$.

Throughout this paper we use (SOT) to denote the strong operator topology.

Theorem 2. Suppose $(X, Y)$ is a multiplier pair and $T$ is a closed densely defined operator on $X$ commuting with every operator in $\mathcal{R}$ and such that $T \subseteq L_{y}$ for some $y \in Y$. Suppose also that, for some $g \in \mathcal{D}(T)$ and for every $x \in X$, there exist $\left\{u_{n}\right\},\left\{v_{n}\right\}$ in $\mathcal{R}_{0}$ such that

(1) $x u_{n}=g v_{n} \in \mathcal{D}(T) \subseteq \mathcal{D}\left(L_{y}\right)$,

(2) $R_{u_{n}} \rightarrow 1$ (SOT).

Then $T=L_{y}$.

Proof. Suppose $x \in \mathcal{D}\left(L_{y}\right)$, so $y x \in X$, and choose $\left\{u_{n}\right\}$ and $\left\{v_{n}\right\}$ as above. Then $x u_{n} \rightarrow x$ and $(y x) u_{n} \rightarrow y x$, but $x u_{n}=g v_{n} \in D(T)$, so $y x u_{n}=T x u_{n}$. Hence $(x, y x)=\lim _{n \rightarrow \infty}\left(x u_{n}, T x u_{n}\right) \in \mathcal{G}(T)^{-}=\mathcal{G}(T)$. Hence $x \in \mathcal{D}(T)$. Thus $\mathcal{D}(T)=\mathcal{D}\left(L_{y}\right)$, so $T=L_{y}$. 
Theorem 3. Suppose $(X, Y)$ is a special multiplier pair such that, for each $y \in Y$, there is a sequence $\left\{q_{n}\right\}$ in $\mathcal{R}_{0}$ such that

(1) $R_{q_{n}} \rightarrow 1$ in the (SOT) on $X$,

(2) $y q_{n} \in \mathcal{R}_{0}$ for every $n$.

If $T$ is a closed densely defined operator on $X$ commuting with $\mathcal{R}$ and there is a $g \in \mathcal{D}(T)$ that is invertible in $Y$, then $T=L_{T(g) g^{-1}}$.

Proof. It follows from condition (1) that $\left\|e-e q_{n}\right\| \rightarrow 0$, which, from the separate continuity of multiplication in $Y$, implies $w-w q_{n}=w \cdot\left(e-e q_{n}\right) \rightarrow 0$ and $w-$ $q_{n} w=\left(e-e q_{n}\right) \cdot w \rightarrow 0$ in $Y$. Hence $\left\{q_{1}, q_{2}, \ldots\right\}$ is both left-separating and rightseparating in $Y$. Suppose $x \in X$ and let $y=g^{-1} x \in Y$. Choose $\left\{q_{n}\right\}$ as above and note that

$$
x q_{n}=g\left(y q_{n}\right),
$$

so, by Theorem 1, we see that $T \subseteq L_{T(g) g^{-1}}$. If $x \in \mathcal{D}\left(L_{T(g) g^{-1}}\right)$, then $x q_{n}=$ $R_{y q_{n}}(g) \in \mathcal{D}(T)$ (since $T$ commutes with $R_{T(g) g^{-1}}$ ), $\left\|x q_{n}-x\right\| \rightarrow 0$ and $T\left(x q_{n}\right)=$ $L_{T(g) g^{-1}}\left(x q_{n}\right)=\left(L_{T(g) g^{-1}} x\right) q_{n} \rightarrow L_{T(g) g^{-1}} x$. Since the operator $T$ is closed, $\left(x, L_{T(g) g^{-1}} x\right)$ is in the graph of $T$. Hence $T=L_{T(g) g^{-1}}$.

We now consider a special case in which $X$ is a Hilbert space and $\mathcal{L}$ and $\mathcal{R}$ are von Neumann algebras.

Lemma 1. Suppose $(X, Y)$ is a multiplier pair, $X$ is a Hilbert space and $\mathcal{L}$ is a von Neumann algebra. Suppose $T$ is a closed densely defined operator affiliated with $\mathcal{L}$. Then there are $u, v \in \mathcal{L}$ with $0 \leq u$ and $\operatorname{ker} u=0$ such that $T \subseteq L_{u^{-1} v}$.

Proof. Since Graph $(T)$ is closed and invariant for the von Neumann algebra $\mathcal{M}=$ $\{A \oplus A: A \in \mathcal{R}\}$, the projection $P$ from $X \oplus X$ onto Graph $(T)$ is in the commutant of $\mathcal{M}$, which is $\mathcal{M}_{2}\left(\mathcal{R}^{\prime}\right)=\mathcal{M}_{2}(\mathcal{L})$. Hence, we can write $P=\left(\begin{array}{ll}A & B \\ C & D\end{array}\right)$ with $A, B, C, D \in \mathcal{L}$. If $\left(\begin{array}{c}x \\ T x\end{array}\right) \in \operatorname{Graph}(T)$, then

$$
\left(\begin{array}{c}
x \\
T x
\end{array}\right)=P\left(\begin{array}{c}
x \\
T x
\end{array}\right)=\left(\begin{array}{c}
A x+B T x \\
C x+D T x
\end{array}\right)
$$

which implies

$$
T x=C x+D T x
$$

or

$$
(1-D) T x=C x .
$$

Suppose $x \in X,\|x\|=1$ and $D x=x$.

Then

$$
\begin{aligned}
\left\|P\left(\begin{array}{l}
0 \\
x
\end{array}\right)\right\|^{2} & =\left(P\left(\begin{array}{l}
0 \\
x
\end{array}\right),\left(\begin{array}{l}
0 \\
x
\end{array}\right)\right)=\left(\left(\begin{array}{c}
B x \\
D x
\end{array}\right),\left(\begin{array}{l}
0 \\
x
\end{array}\right)\right) \\
& =(D x, x)=\left\|\left(\begin{array}{l}
0 \\
x
\end{array}\right)\right\|^{2} .
\end{aligned}
$$

Thus $P\left(\begin{array}{l}0 \\ x\end{array}\right)=\left(\begin{array}{l}0 \\ x\end{array}\right)$, which means $\left(\begin{array}{l}0 \\ x\end{array}\right) \in \operatorname{Graph}(T)$, which, in turn, implies $x=T 0=0$. Thus $\operatorname{ker}(1-D)=0$. We have from $(1-D) T x=C x$ that $T x=$ $(1-D)^{-1} C x$ for every $x \in \mathcal{D}(T)$. 
We now look at a case that will apply to operator algebras with a strictly cyclic separating vector. Suppose $X$ is a Banach space and $\mathcal{A}$ is a norm closed unital subalgebra of $B(X)$ and suppose $0 \neq e \in X$ satisfies

(1) $\mathcal{A} e=X$ (i.e., $e$ is a strictly cyclic vector for $\mathcal{A}$ ),

(2) $e$ is a separating vector for $\mathcal{A}$, i.e., for every $A \in \mathcal{A}$,

$$
A e=0 \Longrightarrow A=0 \text {. }
$$

We can define a multiplication $\cdot$ on $X$ by

$$
(A e) \cdot(B e)=(A B) e .
$$

In this case if we let $X=Y$, we have $(X, Y)$ as a multiplier pair with $\mathcal{L}_{0}=\mathcal{R}_{0}=X$.

Conversely, suppose $(X, Y)$ is a multiplier pair with $\mathcal{L}_{0}=\mathcal{R}_{0}=X$. Then $e$ is a strictly cyclic separating vector for $\mathcal{L}=\left\{L_{x}: x \in \mathcal{L}_{0}\right\}$, since $\mathcal{L} e=\mathcal{L}_{0}=X$ and since $L_{x} e=0$ implies $x e=x=0$.

Proposition 1. Suppose $(X, Y)$ is a multiplier pair and $\mathcal{L}_{0}=\mathcal{R}_{0}=X$. Suppose $T$ is a closed densely defined operator on $X$ affiliated with $\mathcal{L}$. Then $T \in \mathcal{L}$.

Proof. The map $\gamma: \mathcal{L} \rightarrow X$ defined by $\gamma\left(L_{x}\right)=x$ is continuous and bijective. It follows from the open mapping theorem that $\gamma^{-1}$ is continuous. Since $\mathcal{D}(T)$ is dense in $X$, there is a $g \in \mathcal{D}(T)$ with $\|e-g\|<1 /\left\|\gamma^{-1}\right\|$, which means that $\left\|1-L_{g}\right\|=$ $\left\|\gamma^{-1}(e-g)\right\|<1$. Hence $L_{g}$ is invertible in $B(X)$ and in the commutant of $\mathcal{R}$. Hence there is an $h \in \mathcal{L}_{0}$ such that $\eta=h g=e$. It follows from the fact that $\mathcal{D}(T) \subseteq \mathcal{R}(\mathcal{D}(T))$ that $\mathcal{D}(T)=X$, which implies $T \in \mathcal{R}^{\prime}=\mathcal{L}$.

We conclude with some results in a different direction.

Proposition 2. Suppose $(X, Y)$ is a multiplier pair. Then:

(1) If $a \in X$, then $L_{a}$ is a closed densely defined operator on $X$ that commutes with every operator in $\mathcal{R}$.

(2) If $(X, Y)$ is a special multiplier pair and $y \in Y$, then $L_{y}$ is a closed operator on $X$ that commutes with every operator in $\mathcal{R}$.

(3) If $(X, Y)$ is a special multiplier pair, $y \in Y$ and, for each $x \in \mathcal{R}_{0}$, there is a sequence $\left\{e_{n}\right\}$ in $\mathcal{L}_{0}$ such that

(a) $\left\|e_{n} x-x\right\| \rightarrow 0$,

(b) $y e_{n} \in X$ for every $n \geq 1$,

then $\mathcal{D}\left(L_{y}\right)$ is dense in $X$.

Proof. (1) This was proved in [6].

(2) If $\left\{\left(x_{n}, y x_{n}\right)\right\}$ is a sequence in $\operatorname{Graph}\left(L_{y}\right)$ and $\left\|\left(x_{n}, y x_{n}\right)-(x, w)\right\| \rightarrow 0$, then $\left\|x_{n}-x\right\| \rightarrow 0$ and $\left\|y x_{n}-w\right\| \rightarrow 0$. Thus $x_{n} \rightarrow x$ and $y x_{n} \rightarrow w$ in $Y$. Since $(X, Y)$ is a special multiplier pair, multiplication is separately continuous, so $y x_{n} \rightarrow y x$ in $Y$. This implies $w=y x$. Thus $L_{y}$ is closed. Since $(Y,+, \cdot)$ is a ring, $L_{y}$ is in the commutant of $\mathcal{R}$.

(3) It is clear from conditions (a) and (b) that the closure of $\mathcal{D}\left(L_{y}\right)$ contains $\mathcal{R}_{0}$, so $\mathcal{D}\left(L_{y}\right)$ is dense in $X$. 


\section{Applications}

We now apply the results of the preceding section to some special cases of multiplier pairs.

3.1. Analytic functions. Suppose $1 \leq p<\infty$ and $X=H^{p}$ (on the unit disk) and that $Y=N$ is the set of meromorphic functions in the Nevanlinna class, i.e., functions of the form $f / g$ with $f, g \in H^{\infty}$ and $g$ not identically 0 . Then $(X, Y)$ is a special multiplier pair with $\mathcal{L}_{0}=\mathcal{R}_{0}=H^{\infty}$. The Smirnov class $N^{+}$consists of all members of $N$ having a denominator that is an outer function. Sarason [13] has observed that the closed densely defined operators that commute with the unilateral shift on $H^{2}$ are multiplications induced by members of the Smirnov class, and we prove it here more generally in Theorem 4 as a consequence of Corollary 1 . Sarason also observed [14 that members of $N^{+}$have a canonical form related to $H^{2}$, and the following lemma shows that the analogous result related to $H^{p}$ holds for $1 \leq p<\infty$ and is established by the same proof.

Lemma 2. If $1 \leq p<\infty, \phi \in N$, and $\phi \neq 0$, then there exist relatively prime inner functions $u$ and $v$ and outer functions $a$ and $b$ satisfying $|a|^{p}+|b|^{p}=1$ a.e. on the unit circle such that

$$
\phi=\frac{v b}{u a} .
$$

Proof. Recall that an outer function is positive at zero and is uniquely determined by its absolute boundary values, which are necessarily absolutely log integrable. Suppose $\phi$ is a nonzero function in $N$ and the inner-outer factorization is applied to each of the numerator and denominator of $\phi$, so

$$
\phi=\frac{u f_{1}}{v f_{2}},
$$

where $u$ and $v$ are relatively prime inner functions and $f_{1}$ and $f_{2}$ are outer functions in $H^{\infty}$.

Observe that on the unit circle $\mathbb{T}$,

$$
\max \left\{\left|f_{1}\right|,\left|f_{2}\right|\right\} \leq\left(\left|f_{1}\right|^{p}+\left|f_{2}\right|^{p}\right)^{1 / p} \leq\left|f_{1}\right|+\left|f_{2}\right|,
$$

and therefore $\left(\left|f_{1}\right|^{p}+\left|f_{2}\right|^{p}\right)^{1 / p}$ is log integrable. Thus there exists an outer function $\psi$ in $H^{\infty}$ such that $|\psi|=\left(\left|f_{1}\right|^{p}+\left|f_{2}\right|^{p}\right)^{1 / p}$ a.e. on $\mathbb{T}$. Put $a=f_{2} / \psi$ and $b=f_{1} / \psi$ and observe that the definition of $\psi$ implies that $|a|^{p}+|b|^{p}=1$ a.e. on $\mathbb{T}$. The asserted representation of $\phi$ follows.

Corollary 3. If $\phi \in N$, where $\phi=(v b) /(u a)$ as in Lemma 2, then the graph $\operatorname{Graph}\left(M_{\phi}\right)$ of $M_{\phi}$ is the closed subset $\left\{(\mathrm{uag}) \oplus(\mathrm{vbg}): g \in H^{p}\right\}$ of $H^{p} \oplus H^{p}$.

Proof. If $g \in H^{p}$, then $u a g \in H^{p}$ and $M_{\phi} u a g=v b g \in H^{p}$, and it follows that the set asserted to be the graph of $M_{\phi}$ is a subset of the graph. For the opposite inclusion suppose both $f$ and $\phi f$ belong to $H^{p}$. Then both $|f|^{p}$ and $|\phi|^{p}|f|^{p}$ are integrable on $\mathbb{T}$, and because

$$
\frac{|f|^{p}}{|a|^{p}}=\frac{|a|^{p}+|b|^{p}}{|a|^{p}}|f|^{p}=|f|^{p}+|\phi|^{p}|f|^{p}
$$

on $\mathbb{T}$, it follows that if $g_{1}=f / a$, then $g_{1} \in H^{p}$. (If $f \in H^{p}, a$ is outer, and $f / a \in L^{p}$, then $f / a \in H^{p}$. See Nikolskii [12, Theorem 3.9.6].) Thus $f=a g_{1}$ and $u \phi f=v b g_{1}$. Since $u$ and $v$ are relatively prime and $b$ is outer, the last equation 
shows that $u$ is a factor of $g_{1}$, and thus $g_{1}=u g$ for some $g \in H^{p}$. We have shown that $f=a u g$ and $\phi f=v b g$, and thus the required inclusion is established.

Theorem 4. Suppose $1 \leq p<\infty$ and $\mathcal{G} \subseteq H^{p} \oplus H^{p}$ is a graph that is invariant under $M_{z} \oplus M_{z}$. Then there is a meromorphic $\phi \in N$ such that $\mathcal{G} \subseteq \operatorname{Graph}\left(M_{\phi}\right)$. If the domain of $\mathcal{G}$ is dense in $H^{p}$, then $\phi$ is in the Smirnov class. If, in addition, $\mathcal{G}$ is closed, then $\phi$ is in the Smirnov class and $\mathcal{G}=\operatorname{Graph}\left(M_{\phi}\right)$.

Proof. The first assertion follows from Corollary 1. Suppose the domain $\mathcal{D}(\mathcal{G})$ of $\mathcal{G}$ is dense. Then for every $z$ with $|z|<1$, there is a $g \in \mathcal{D}(\mathcal{G})$ such that $g(z) \neq 0$, so $\phi g \in H^{p}$ implies that $\phi$ has a removable singularity at $z$. Hence $\phi$ is analytic on the open unit disk. By Corollary 3 , the domain of $M_{\phi}$, which includes that of $\mathcal{G}$, is $u a H^{p}$. Thus $u a H^{p}$ is dense, and it follows that $u=1$. Thus $\phi$ is in $N^{+}$.

Suppose that $\mathcal{G}$ is closed. If $H^{p} \oplus H^{p}$ is given the norm defined by $\|f \oplus g\|=$ $\left(\|f\|_{p}^{p}+\|g\|_{p}^{p}\right)^{1 / p}$, then the proof of Corollary 3 shows that the map $V: H^{p} \rightarrow$ $H^{p} \oplus H^{p}$ defined by

$$
V g=u a g \oplus v b g
$$

is an isometry of $H^{p}$ onto $\operatorname{Graph}\left(M_{\phi}\right)$. Let $\mathcal{M}$ be the inverse image of $\mathcal{G}$ under $V$. Then $\mathcal{G}$ is a closed subspace of $H^{p}$, and for $g \in \mathcal{M}$ we have

$$
V M_{z} g=u a z g \oplus v b z g=M_{z} \oplus M_{z} V g \in \mathcal{G} .
$$

Hence $\mathcal{M}$ is invariant under $M_{z}$, and, by Duren [4, Theorem 7.4] or Helson [10, page 25], $\mathcal{M}=\omega H^{p}$ for some inner function $\omega$, and thus

$$
\mathcal{G}=\left\{\omega u a g \oplus \omega v b g: g \in H^{p}\right\}=\left(M_{\omega} \oplus M_{\omega}\right) \operatorname{Graph}\left(M_{\phi}\right) .
$$

It follows that if the domain of $\mathcal{G}$ is dense, then $\omega$, as well as $u$, must be 1 , and hence the asserted equality holds.

As a corollary to the proof we have the following.

Corollary 4. If $1 \leq p<\infty$ and $\mathcal{G} \subseteq H^{p} \oplus H^{p}$ is a closed graph that is invariant under $M_{z} \oplus M_{z}$, then there is a meromorphic function $\phi$ in the Nevanlinna class and an inner function $\omega$ such that

$$
\mathcal{G}=\left(M_{\omega} \oplus M_{\omega}\right) \operatorname{Graph}\left(M_{\phi}\right) .
$$

The following is a direct consequence of the preceding corollary (i.e., $\omega$ is constant).

Corollary 5. Suppose $1 \leq p<\infty$ and $\mathcal{G} \subseteq H^{p} \oplus H^{p}$ is a closed graph that is invariant under $M_{z} \oplus M_{z}$ and the sum of its domain and range is dense in $H^{p}$. Then it is the graph of some meromorphic function in the Nevanlinna class.

3.2. Measure theory. A symmetric norm on $L^{\infty}[0,1]$, with respect to Lebesgue measure $\mu$, is a norm $\alpha$ such that

(1) $\alpha(f)=\alpha(|f|)$ for every $f \in L^{\infty}[0,1]$,

(2) $\alpha(1)=1$,

(3) $\alpha(f \circ \tau)=\alpha(f)$ for every $f \in L^{\infty}[0,1]$ and every invertible measurepreserving $\tau:[0,1] \rightarrow[0,1]$ 
We say that a symmetric norm $\alpha$ on $L^{\infty}[0,1]$ is continuous if

$$
\lim _{t \rightarrow 0^{+}} \alpha\left(\chi_{[0, t]}\right)=0 \text {. }
$$

We define $L^{\alpha}[0,1]$ to be the completion of $L^{\infty}[0,1]$ with respect to the norm $\alpha$. We can realize the elements of $L^{\alpha}[0,1]$ as (equivalence classes of) measurable functions in $L^{1}[0,1]$. Moreover, if $X=L^{\alpha}[0,1]$ and $Y$ is the set of all measurable functions on $[0,1]$ with the topology of convergence in measure, then $(X, Y)$ is a special multiplier pair with pointwise multiplication, and $\mathcal{R}_{0}=\mathcal{L}_{0}=L^{\infty}[0,1]$ (see [7]). Moreover, if $\alpha$ is continuous, we have

$$
\lim _{\mu(F) \rightarrow 0} \alpha\left(\chi_{F} h\right)=0
$$

for every $h \in L^{\alpha}[0,1]$.

Proposition 3. Suppose $\alpha$ is a continuous symmetric norm on $L^{\infty}[0,1]$ and $\mathcal{G} \subseteq$ $L^{\alpha}[0,1] \oplus L^{\alpha}[0,1]$ is a closed linear subspace and a graph that is invariant under $M_{f} \oplus M_{f}$ for every $f \in L^{\infty}[0,1]$. Then there is a measurable function $\varphi$ (with possibly infinite values) such that $\mathcal{G} \subseteq \mathcal{G}\left(M_{\varphi}\right)$.

Proof. Since $\mathcal{D}(\mathcal{G})$ is an $L^{\infty}[0,1]$-module, we have $|g| \in \mathcal{D}(\mathcal{G})$ whenever $g \in \mathcal{D}(\mathcal{G})$. Moreover, if $\left\{g_{n}\right\}$ is a sequence in $\mathcal{D}(\mathcal{G})$ and $\left(\left|g_{n}\right|, h_{n}\right) \in \mathcal{G}$ for $n \geq 1$, then $g=\sum_{n=1}^{\infty} t_{n}\left|g_{n}\right| \in \mathcal{D}(\mathcal{G})$, where $0<t_{n}<\frac{1}{2^{n}\left[\alpha\left(g_{n}\right)+\alpha\left(h_{n}\right)+1\right]}$ for $n \geq 1$. Since $\{x: g(x)>0\}=\bigcup_{n \geq 1}\left\{x:\left|g_{n}(x)\right|>0\right\}$, if we choose $g_{n}$ so that

$$
\mu\left(\left\{x: g_{n}(x) \neq 0\right\}\right)>\sup \{\mu(\{x: h(x) \neq 0\}): h \in \mathcal{D}(\mathcal{G})\}-\frac{1}{n},
$$

then we see that

$$
\mu(\{x: g(x) \neq 0\})=\sup \{\mu(\{x: h(x) \neq 0\}): h \in \mathcal{D}(\mathcal{G})\} .
$$

If $E=\{x: g(x) \neq 0\}$, then $\chi_{E} h=h$ a.e. $(\mu)$ for every $h \in \mathcal{D}(\mathcal{G})$, and, since $\mathcal{G}$ is a graph and an $L^{\infty}[0,1]$-module, we see that $\chi_{E} h=h$ a.e. $(\mu)$ for every $h$ in the range of $\mathcal{G}$. We can now replace $L^{\alpha}[0,1]$ with $\chi_{E} L^{\alpha}[0,1]=X$ and let $Y$ be the set of measurable functions that vanish on $[0,1] \backslash E$. Now $g$ has a multiplicative inverse, and, for each $h \in X$ and each positive integer $n \geq 1$, we have $h \chi_{E_{n}}=g\left(\chi_{E_{n}} h / g\right)$, where $E_{n}=\{x: g(x) \geq 1 / n$ and $|f(x)| \leq n\}$. Since $\bigcup_{n \geq 1} E_{n}=E$ (a.e.), the set $\left\{\chi_{E_{n}}: n \geq 1\right\}$ is left separating for $Y$. Applying Theorem 1 to $\chi_{E} \mathcal{G}$, we see that

$$
\mathcal{G}=\chi_{E} \mathcal{G} \subseteq L_{f / g}
$$

where $(g, f) \in \mathcal{G}$. Moreover, the fact that $\mu\left(E \backslash E_{n}\right) \rightarrow 0$ and $\alpha$ is continuous implies

$$
R_{\chi_{E_{n}}} \rightarrow 1
$$

in the strong operator topology. It follows from Theorem 2 that $\chi_{E} \mathcal{G}$ is the graph of $L_{f / g}$ on $\chi_{E} L^{\alpha}[0,1]$. If we let $\varphi=\frac{f}{g} \chi_{E}+\infty \chi_{([0,1] \backslash E)}$, we see that $\mathcal{G}=\mathcal{G}\left(L_{\varphi}\right)$.

Corollary 6. Suppose $\alpha$ is a continuous symmetric norm on $L^{\infty}[0,1]$ and $T$ is a closed densely defined linear transformation on $L^{\alpha}[0,1]$ that commutes with $L_{f}$ for every $f \in L^{\infty}[0,1]$. Then there is a measurable function $\varphi:[0,1] \rightarrow \mathbb{C}$ such that $T=L_{\varphi}$ 
Theorem 5. Suppose $\alpha$ is a symmetric norm on $L^{\infty}[0,1]$ and $T$ is a closed densely defined operator on $L^{\alpha}[0,1]$ that commutes with $M_{x}$. Then there is a measurable function $\varphi:[0,1] \rightarrow \mathbb{C}$ such that $T=M_{\varphi}$.

Proof. If $\alpha$ is continuous, the desired conclusion follows from Corollary 6, If $\alpha$ is not continuous, then $\alpha$ is equivalent to $\|\cdot\|_{\infty}$ on $L^{\infty}[0,1]$ (see [7]), so $L^{\alpha}[0,1]=$ $L^{\infty}[0,1]$, and the desired conclusion follows from Proposition 1$]$

3.3. Finite von Neumann algebras. Suppose $\mathcal{M}$ is a $I I_{1}$ factor von Neumann algebra with a faithful normal tracial state $\tau$. A unitarily invariant norm on $\mathcal{M}$ is a norm $\beta$ such that

(1) $\beta(U T V)=\beta(T)$ for all $U, T, V \in \mathcal{M}$ with $U, V$ unitary,

(2) $\beta(1)=1$.

Since $\mathcal{M}$ is a $I I_{1}$ factor, there is a chain $\left\{P_{t}: t \in[0,1]\right\}$ of projections in $\mathcal{M}$ such that $\tau\left(P_{t}\right)=t$ for every $t \in[0,1]$. The map $\chi_{[0, t]} \mapsto P_{t}$ extends to a unital *-homomorphism $\pi: L^{\infty}[0,1] \rightarrow \mathcal{M}$ such that

$$
\tau(\pi(f))=\int_{0}^{1} f(x) d x
$$

for every $f \in L^{\infty}[0,1]$. If we define $\alpha: L^{\infty}[0,1] \rightarrow[0, \infty)$ by

$$
\alpha(f)=\beta(\pi(f)),
$$

we obtain a symmetric norm $\alpha$. It turns out that $\alpha$ is independent of the chain $\left\{P_{t}: t \in[0,1]\right\}$. Moreover, every element $A \in \mathcal{M}$ has a polar decomposition $A=$ $U\left(A^{*} A\right)^{1 / 2}$ and $\beta(A)=\beta\left(\left(A^{*} A\right)^{1 / 2}\right)$. There is a chain $\left\{P_{t}: t \in[0,1]\right\}$ whose generated von Neumann algebra contains $\left(A^{*} A\right)^{1 / 2}$, and there is a $\varphi \in L^{\infty}[0,1]$ such that $\pi(\varphi)=\left(A^{*} A\right)^{1 / 2}$, and we get $\beta(A)=\alpha(\varphi)$. In this way we can recapture $\beta$ from $\alpha$. It is shown in [5] that every symmetric norm $\alpha$ on $L^{\infty}[0,1]$ determines a unitarily invariant norm $\beta$ on $\mathcal{M}$, and we will call this norm $\|\cdot\|_{\alpha}$. We let $L^{\alpha}(\mathcal{M}, \tau)$ denote the completion of $\mathcal{M}$ with respect to the norm $\|\cdot\|_{\alpha}$. If the symmetric norm $\alpha$ is continuous, then

$$
\lim _{\tau(P) \rightarrow 0, P=P^{*}=P^{2} \in \mathcal{M}}\|P\|_{\alpha}=0 .
$$

If $\alpha$ is not continuous, then $\alpha$ is equivalent to $\|\cdot\|_{\infty}$ on $L^{\infty}[0,1]$ and $\|\cdot\|_{\alpha}$ is equivalent to the operator norm on $\mathcal{M}$, so $L^{\beta}(\mathcal{M}, \tau)=\mathcal{M}$.

There is a topology of convergence in measure on $\mathcal{M}$ (see [11]), and $L^{\alpha}(\mathcal{M}, \tau)$ can be viewed as a subset of the completion $\mathcal{Y}$ of $\mathcal{M}$ with respect to this topology. It was shown in [7] that $\left(L^{\alpha}(\mathcal{M}, \tau), \mathcal{Y}\right)$ is a multiplier pair and $\mathcal{L}_{0}=\mathcal{R}_{0}=\mathcal{M}$, and it follows from [11, Theorem 1] that $\left(L^{\alpha}(\mathcal{M}, \tau), \mathcal{Y}\right)$ is a special multiplier pair.

Theorem 6. Suppose $\mathcal{M}$ is a $I I_{1}$ factor von Neumann algebra with faithful normal tracial state $\tau$, and suppose $\alpha$ is a unitarily invariant norm on $\mathcal{M}$. Suppose $T$ is a closed densely defined operator on $L^{\alpha}(\mathcal{M}, \tau)$ that commutes with $R_{S}$ for every $S \in \mathcal{M}$. Then:

(1) If $\alpha$ is not continuous, then $T \in \mathcal{L}$.

(2) If $\alpha$ is continuous, then $T=L_{y}$ for some $y \in \mathcal{Y}$.

(3) If $\alpha$ is continuous and $y \in \mathcal{Y}$, then $L_{y}$ is a closed densely defined operator on $L^{\alpha}(\mathcal{M}, \tau)$ that commutes with $R_{S}$ for every $S \in \mathcal{M}$. 
Proof. (1) If $\alpha$ is not continuous, then $\alpha$ is equivalent to the operator norm on $\mathcal{M}$, so $L^{\alpha}(\mathcal{M}, \tau)=\mathcal{M}$, which implies $\mathcal{L}_{0}=\mathcal{R}_{0}=\mathcal{M}$. The desired conclusion now follows from Proposition 1 .

(2) Suppose $x \in \mathcal{D}(T)$. It follows from [11, Theorem 2] that there is a sequence $\left\{p_{n}\right\}$ of projections in $\mathcal{M}$ such that $\tau\left(p_{n}\right) \rightarrow 0$ and $x\left(1-p_{n}\right) \in \mathcal{M}$ for each $n \geq 1$, and, since $\mathcal{D}(T)\left(1-p_{n}\right) \subseteq \mathcal{D}(T)$, we have $x\left(1-p_{n}\right) \in \mathcal{M} \cap \mathcal{D}(T)$ for each $n \geq 1$. However, $\alpha$ is continuous, so

$$
\alpha\left(x-\left(1-p_{n}\right) x\right)=\alpha\left(p_{n} x\right) \leq \alpha\left(p_{n}\right)\|x\| \rightarrow 0 .
$$

Thus the closure of $\mathcal{M} \cap \mathcal{D}(T)$ contains $\mathcal{D}(T)$, and since $\mathcal{D}(T)$ is dense in $L^{\alpha}(\mathcal{M}, \tau)$, it follows that $\mathcal{M} \cap \mathcal{D}(T)$ is dense in $L^{\alpha}(\mathcal{M}, \tau)$. For each $x \in \mathcal{M} \cap \mathcal{D}(T)$, let $P_{x}$ denote the projection onto the weak operator topology closure of the range of $x$, and choose a sequence $\left\{x_{n}\right\}$ in $\mathcal{M} \cap \mathcal{D}(T)$ such that

$$
\lim \tau\left(P_{x_{n}}\right)=\sup \left\{\tau\left(P_{x}\right): x \in \mathcal{M} \cap \mathcal{D}(T)\right\} .
$$

For each $n \geq 1$ we can write $x_{n}=\left(x_{n} x_{n}^{*}\right)^{\frac{1}{2}} u_{n}$ with $u_{n}$ unitary, and so $\left(x_{n} x_{n}^{*}\right)^{\frac{1}{2}}=$ $R_{u_{n}^{*}} x_{n} \in \mathcal{M} \cap \mathcal{D}(T)$. If we choose $t_{n}$ so that

$$
t_{n}\left[\alpha\left(\left(x_{n} x_{n}^{*}\right)^{\frac{1}{2}}\right)+\alpha\left(T\left(x_{n} x_{n}^{*}\right)^{\frac{1}{2}}\right)\right]<\frac{1}{2^{n}},
$$

then $g=\sum_{n=1}^{\infty} t_{n}\left(x_{n} x_{n}^{*}\right)^{\frac{1}{2}} \in \mathcal{M} \cap \mathcal{D}(T)$. Moreover, it follows that $P_{g} \geq P_{\left(x_{n} x_{n}^{*}\right)^{\frac{1}{2}}}$ for each $n$. Hence

$$
\tau\left(P_{g}\right)=\sup \left\{\tau\left(P_{x}\right): x \in \mathcal{M} \cap \mathcal{D}(T)\right\} .
$$

If $x \in \mathcal{D}(T)$, then $\left(x x^{*}\right)^{\frac{1}{2}}, g+\left(x x^{*}\right)^{\frac{1}{2}} \in \mathcal{D}(T)$, which means the weak operator topology closure of the range of $x$ (which equals the range of $\left(x x^{*}\right)^{\frac{1}{2}}$ ) is contained in $P_{g}$. Hence,

$$
\mathcal{M} \cap \mathcal{D}(T) \subseteq P_{g} \mathcal{M} \subseteq P_{g} L^{\alpha}(\mathcal{M}, \tau) .
$$

Since $\mathcal{M} \cap \mathcal{D}(T)$ is dense in $L^{\alpha}(\mathcal{M}, \tau)$, we must have $P_{g}=1$, which implies $g$ is invertible in $\mathcal{Y}$.

Now it follows from [11, Theorem 2] that for each $y \in \mathcal{Y}$ there is a sequence $\left\{q_{n}\right\}$ of projections in $\mathcal{M}$ such that $\tau\left(q_{n}\right) \rightarrow 1$ and $y q_{n} \in \mathcal{M}$ for every $n \geq 1$. Since $\alpha$ is continuous, we know from [5] that $R_{q_{n}} \rightarrow 1$ (SOT) on $L^{\alpha}(\mathcal{M}, \tau)$. Hence, Theorem 3 implies that $T=L_{T(g) g^{-1}}$.

(3) It follows from [6] that $L_{y}$ is a closed operator on $L^{\alpha}(\mathcal{M}, \tau)$. It follows from [11, Theorem 2] that there is a sequence $\left\{p_{n}\right\}$ of projections in $\mathcal{M}$ such that $\tau\left(p_{n}\right) \rightarrow 0$ and $y\left(1-p_{n}\right) \in \mathcal{M} \subseteq L^{\alpha}(\mathcal{M}, \tau)$, which implies

$$
\bigcup_{n=1}^{\infty}\left(1-p_{n}\right) \mathcal{M} \subseteq \mathcal{D}\left(L_{y}\right) .
$$

Moreover, since $\alpha$ is continuous,

$$
\alpha\left(x-\left(1-p_{n}\right) x\right)=\alpha\left(p_{n} x\right) \leq \alpha\left(p_{n}\right)\|x\| \rightarrow 0 .
$$

Hence, $\mathcal{D}\left(L_{y}\right)$ is dense in $L^{\alpha}(\mathcal{M}, \tau)$. 


\section{An example on the Bergman space}

The closed densely defined operators commuting with the unilateral shift, $M_{z}$ on $H^{2}$, are multiplications by functions in the Smirnov class. Is there a similar result for the Bergman shift, multiplication by $z$ on the Bergman space $A^{2}$ ? What follows is a counterexample.

Theorem 7. There exists a closed densely defined operator that commutes with the Bergman shift but is not a multiplication operator.

Proof. By a result of Seip (see [16], 3, Theorem 9, p. 186], 8]) there exist two disjoint interpolation sets $\Gamma_{1}, \Gamma_{2}$ for $A^{2}$ whose union $\Gamma$ is a sampling set. A sampling set for $A^{2}$ is defined as a subset $\Gamma$ of $\mathbb{D}$ with the property that there exist positive constants $c$ and $C$ such that, for each $f \in A^{2}$,

$$
c\|f\|^{2} \leqslant \sum_{z \in \Gamma}|f(z)|^{2}\left(1-|z|^{2}\right)^{2} \leqslant C\|f\|^{2} .
$$

Thus, with $\mu$ the discrete measure having mass $\left(1-|z|^{2}\right)^{2}$ at each point $z$ of $\Gamma$, the restriction map $\Phi: f \mapsto f \mid \Gamma$ is an invertible map of $A^{2}$ onto a subspace $A^{2} \mid \Gamma$ of $L^{2}(\mu)$. An interpolation set for $A^{2}$ is a subset $\Gamma$ of $\mathbb{D}$ with the property that for each element $w$ in $L^{2}(\mu)$ there exists $f \in A^{2}$ such that $w=\Phi(f)$, i.e., the restriction map $f \mapsto f \mid \Gamma$ on $A^{2}$ is onto $L^{2}(\mu)$.

Since interpolation sets are also zero sets for $A^{2}$, we can let $f_{1}$ be a function in $A^{2}$ having $\Gamma_{1}$ as its zero set. Define $\mathcal{G}_{1}, \mathcal{G}_{2}$ by

$$
\mathcal{G}_{1}=\left\{\left(u, u f_{1}\right): u, u f_{1} \in A^{2}\right\}
$$

and

$$
\mathcal{G}_{2}=\left\{(g, g): g \in A^{2}, g \mid \Gamma_{2}=0\right\} .
$$

Then $\mathcal{G}_{1}=\mathcal{G}\left(M_{f_{1}}\right)$ and $\mathcal{G}_{2} \subseteq \mathcal{G}(I)$ are graphs of closed operators that commute with the Bergman shift $M_{z}$. Moreover, $\mathcal{G}=\mathcal{G}_{1}+\mathcal{G}_{2}$ is also a graph. To see that $\mathcal{G}$ is a graph, we need only check that if $(0, h) \in \mathcal{G}$, then $h=0$. Suppose $\left(u, u f_{1}\right) \in \mathcal{G}_{1}$, $(g, g) \in \mathcal{G}_{2}$ and $(0, h)=\left(u, u f_{1}\right)+(g, g)$. Then $u=-g$ vanishes on $\Gamma_{2}$, so $u f_{1}$ vanishes on the sampling set $\Gamma=\Gamma_{1} \cup \Gamma_{2}$, which implies $u f_{1}=0$. But $f_{1} \neq 0$, so $0=u=-g$, whence $h=0$.

Finally, it will be shown that the angle between $\mathcal{G}_{1}$ and $\mathcal{G}_{2}$ is greater than zero, and thus the graph $\mathcal{G}=\mathcal{G}_{1}+\mathcal{G}_{2}$ is itself a closed direct sum. It is to be shown that there is a $\gamma, 0 \leq \gamma<1$, such that, if $F_{k} \in \mathcal{G}_{k}$ for $k=1,2$, then

$$
\left|\left\langle F_{1}, F_{2}\right\rangle\right| \leq \gamma\left\|F_{1}\right\|\left\|F_{2}\right\| .
$$

Let $\Phi^{(2)}(f, g)=(\Phi(f), \Phi(g))$. Since $\Phi$ is an isomorphism between $A^{2}$ and its image $\mathcal{A}$ in $L^{2}(\mu)$, it suffices to show that the preceding inequality holds for the images of $F_{1}$ and $F_{2}$ under $\Phi^{(2)}$. Since an element of $L^{2}(\mu)$ in the image $\mathcal{A}$ of $\Phi$ is just the restriction of an element of $A^{2}$, we will simply write $f=\Phi(f)$ and indicate the norm and inner product in $L^{2}(\mu)$ with a subscript $\mu$. We have $F_{1}=\left(u, u f_{1}\right)$ and $F_{2}=(g, g)$ with $g, u, u f_{1} \in A^{2}$ and $g \mid \Gamma_{2}=0$. Thus

$$
\left\langle F_{1}, F_{2}\right\rangle_{\mu}=\langle u, g\rangle_{\mu}+\left\langle u f_{1}, g\right\rangle_{\mu}=\langle u, g\rangle_{\mu},
$$


where the second equality follows from the fact that $f_{1} \mid \Gamma_{1}=0$ as well as $g \mid \Gamma_{2}=0$, so $u f_{1} \bar{g}$ vanishes a.e.- $\mu$. Also,

$$
\left\|F_{1}\right\|_{\mu}^{2}=\|u\|_{\mu}^{2}+\left\|u f_{1}\right\|_{\mu}^{2}
$$

and

$$
\left\|F_{2}\right\|_{\mu}^{2}=2\|g\|_{\mu}^{2}
$$

We have

$$
\left|\left\langle F_{1}, F_{2}\right\rangle_{\mu}\right|=\left|\langle u, g\rangle_{\mu}\right|^{2} \leqslant\|u\|_{\mu}\|g\|_{\mu} \leq\left\|F_{1}\right\|_{\mu}(\sqrt{2} / 2)\left\|F_{2}\right\|_{\mu},
$$

as required with $\gamma=\sqrt{2} / 2$. It follows that the subspaces are at a positive angle and the sum of their closures is closed. The operator whose graph is $\mathcal{G}$ has the required properties.

\section{REFERENCES}

[1] Bercovici, Hari, The unbounded commutant of an operator of class $C_{0}$, Oper. Matrices 3 (2009), no. 4, 599-605. MR2603336(2011d:47032)

[2] Bercovici, H.; Douglas, R. G.; Foias, C.; Pearcy, C., Confluent operator algebras and the closability property, J. Funct. Anal. 258 (2010), no. 12, 4122-4153. MR2609540(2012b:47015)

[3] Duren, Peter; Schuster, Alexander, Bergman spaces, Mathematical Surveys and Monographs, no. 100, American Mathematical Society, 2000. MR2033762 (2005c:30053)

[4] Duren, Peter, Theory of $H^{p}$ spaces, Pure and Applied Mathematics, Vol. 38, Academic Press, New York and London, 1970. MR0268655 (42:3552)

[5] Fang, Junsheng; Hadwin, Don; Nordgren, Eric; Shen, Junhao, Tracial gauge norms on finite von Neumann algebras satisfying the weak Dixmier property, J. Funct. Anal. 255 (2008), no. 1, 142-183. MR2417813(2010a:46151)

[6] Hadwin, Don and Nordgren, Eric, A general view of multipliers and composition operators, Linear Algebra Appl. 383 (2004), 187-211. MR2073904 (2005g:47123)

[7] Hadwin, Don and Nordgren, Eric, A general view of multipliers and composition operators II, Banach spaces of analytic functions, 63-73, Contemp. Math., 454, Amer. Math. Soc., Providence, RI, 2008. MR2408235 (2009m:47186)

[8] Hedenmalm, Håkan; Richter, Stefan; Seip, Kristian, Interpolating sequences and invariant subspaces of given index in the Bergman spaces, J. Reine Angew. Math. 477 (1996), 13-30. MR $1405310(97 \mathrm{i}: 46044)$

[9] Kadison, Richard V.; Ringrose, John R., Fundamentals of the theory of operator algebras. Vol. I: Elementary theory. Reprint of the 1983 original. Graduate Studies in Mathematics, 15. American Mathematical Society, Providence, RI, 1997. MR 1468229 (98f:46001a)

[10] Helson, Henry, Lectures on invariant subspaces, Academic Press, New York and London, 1964. MR0171178 (30:1409)

[11] Nelson, Edward, Notes on non-commutative integration, J. Funct. Anal. 15 (1974), 103-116. MR 0355628(50:8102)

[12] Nikolski, Nikolai K., Operators, functions and systems: an easy reading, Vol. 1, Hardy, Hankel, and Toeplitz, translated from the French by Andreas Hartmann. Mathematical Surveys and Monographs, 92. American Mathematical Society, Providence, RI, 2002. MR.1864396 (2003i:47001a)

[13] Sarason, Donald, Unbounded Toeplitz operators, Integral Equations Operator Theory 61 (2008), no. 2, 281-298. MR2418122(2010c:47073)

[14] Sarason, Donald, Unbounded operators commuting with restricted backward shifts, Oper. Matrices 2 (2008), no. 4, 583-601. MR2468883 (2009k:47083)

[15] Sarason, Donald, Unbounded operators commuting with the commutant of a restricted backward shift, Oper. Matrices 4 (2010), no. 2, 293-300. MR2667340(2011d:47054)

[16] Seip, Kristian, Beurling type density theorems in the unit disk, Invent. Math. 113 (1993), 21-39. MR 1223222 (94g:30033) 
[17] Seubert, Steven M., Unbounded dissipative compressed Toeplitz operators, J. Math. Anal. Appl. 290 (2004), 132-146. MR2032231 (2004i:47053)

[18] Suárez, Daniel, Closed commutants of the backward shift operator, Pacific J. Math. 179 (1997), no. 2, 371-396. MR.1452540(99a:47050)

Department of Mathematics, Kingsbury Hall, University of New Hampshire, Durham, New Hampshire 03824-3591

E-mail address: don@math.unh.edu

Department of Mathematics, University of Pennsylvania, 209 South 33rd Street, Philadelphia, Pennsylvania 19104

E-mail address: zheliu@sas.upenn.edu

Department of Mathematics, Kingsbury Hall, University of New Hampshire, Durham, New HAMPSHIRE 03824-3591

E-mail address: ean@math.unh.edu 